\title{
Intraoperative Hypothermia During Cytoreductive Surgery for Ovarian Cancer and Perioperative Morbidity
}

\author{
Mehdi Moslemi-Kebria, MD, Sherif A. El-Nashar, MBBCh, MS, Giovanni D. Aletti, MD, \\ and William A. Cliby, MD
}

OBJECTIVE: To evaluate intraoperative hypothermia as a predictor for morbidity after open abdominal surgery for ovarian cancer.

METHODS: This cohort study included 146 women with stage IIIC and IV ovarian cancer who underwent debulking surgery at our institution from January 1, 2001, through December 31, 2003. Hypothermia was defined as end operative temperature lower than $36^{\circ} \mathrm{C}$. Early complications (occurring within 30 days of surgery) included: mortality, infectious morbidities, cardiovascular events, venous thromboembolic (VTE) events, anastomotic leak, readmission, and reoperation. Survival was also evaluated. Logistic regression models were used to adjust for known confounders.

RESULTS: The mean age was $63.9 \pm 11.7$ years; 46 (32\%) patients had a body mass index higher than 30 ; mean operative time was $239 \pm 85$ minutes. There were five deaths perioperatively, all in the hypothermic group. Hypothermia was associated with an increased risk of any early complications (34 [42.0\%] compared with 11 [16.9\%]) with an adjusted odds ratio (OR) of 3.40 (95\% confidence interval [CI] 1.48-8.33). For individual complications, hypothermic patients were at higher risk for VTE events with an adjusted OR of 3.53 (95\% Cl 1.0216.44); infectious morbidity with an adjusted OR of 2.99 (95\% Cl 0.97-11.35); and reoperation with an adjusted OR of 4.96 (95\% Cl $0.80-95.7)$. The overall survival was

$\overline{\text { From the Divisions of Gynecology Surgery, Mayo Clinic, Rochester, Minnesota; }}$ and the Department of Obstetrics and Gynecology, Gynecological Oncology Service, Cleveland Clinic, Cleveland, Ohio.

Presented at the Society of Gynecologic Oncology 41st Annual Meeting on Women's Cancer, March 14-17, 2010, San Francisco, California.

Corresponding author: William A. Cliby, MD, Division of Gynecologic Surgery, Mayo Clinic, 200 First Street SW, Rochester, MN 55905; e-mail: cliby.william@mayo.edu.

Financial Disclosure

The authors did not report any potential conflicts of interest.

(C) 2012 by The American College of Obstetricians and Gynecologists. Published by Lippincott Williams E Wilkins.

ISSN: 0029-7844/12 shorter in hypothermic group with a median of 34 compared with 45 months $(P=.045)$; this remained significant for an optimally resected subgroup with a median overall survival of 40 compared with 48 months $(P=.049)$.

CONCLUSION: Surgical hypothermia is an independent predictor of early perioperative complications and overall survival after cytoreductive surgery for ovarian cancer. This is a critically important finding, because maintaining normothermia is an inexpensive modifiable factor, which could result in reduced morbidity.

LEVEL OF EVIDENCE: II

(Obstet Gynecol 2012;119:590-6)

DOI: 10.1097/AOG.0b013e3182475f8a

$\mathrm{P}$ erioperative hypothermia is common in patients undergoing major open abdominal surgery. Visceral and peritoneal surface exposure to room temperature and anesthetic-induced impairment of thermoregulatory control are the most accepted etiologies of intraoperative hypothermia. ${ }^{1}$ Patients undergoing cytoreductive surgery for ovarian cancer are at high risk of intraoperative hypothermia as a result of the previously mentioned factors combined with long operative times with long incisions and large intravenous fluid requirements. Ascites production, its removal, and evaporation during surgery may also contribute to lowering body temperature. Most published literature defines operative hypothermia as a core body temperature of less than $36^{\circ} \mathrm{C} .^{2-4}$ Studies have shown that hypothermia is associated with increased perioperative blood loss and higher blood transfusion rates; it is also associated with increased rates of surgical wound infection, prolonged hospitalization, and increased incidence of postoperative adverse myocardial events. ${ }^{2-4}$

The overall rate of complications after cytoreduction for ovarian cancer is at least 20\% dependent on risk factors and surgical complexity. ${ }^{5}$ Among the 
potential predisposing factors for postoperative complications, hypothermia is one that can be prevented or minimized with active warming measures during surgery. To examine the possible effect of intraoperative hypothermia in patients with advanced ovarian cancer undergoing cytoreductive surgery, we performed a retrospective study and tested the hypothesis that intraoperative hypothermia is associated with a greater incidence of perioperative complications.

\section{MATERIALS AND METHODS}

The Mayo Clinic institutional review board reviewed and approved this cohort study. The article was written in accordance with the Strengthening the Reporting of Observational Studies in Epidemiology statement. ${ }^{6}$ Only those patients who approved the use of their medical records for research were included in the study.

The cohort of patients diagnosed with primary epithelial ovarian cancer between January 1, 2001, and December 31, 2003, was identified from surgical records using the electronic medical records linkage system at Mayo Clinic. Only patients undergoing primary surgical exploration with a postoperative diagnosis of stage IIIC or IV epithelial ovarian cancer were included. To obtain a homogeneous population undergoing similar cytoreductive surgeries for comparisons, patients with disease limited to the pelvis with retroperitoneal involvement only (upstaged to IIIC disease solely by virtue of lymph nodal involvement) were excluded from the analysis because they require less extensive operations and have a significantly better prognosis. ${ }^{5}$ Preoperative medical evaluations were performed for all patients, and each was classified by American Society of Anesthesiologists score. All patients were scheduled for treatment with intravenous first-line postoperative platinum-based chemotherapy.

All patients underwent exploratory laparotomy with attempted maximal cytoreduction. All patients underwent active warming during surgery, predominantly with an upper body forced-air warmer. Fluid warmers were used at the discretion of the anesthesiologists, and all patients received perioperative antibiotics. All surgeries were performed by experienced gynecologic oncologists at Mayo Clinic using similar teams, perioperative care, and a standardized surgical strategy. Perioperative variables such as end operative temperature, age, body mass index (BMI, calculated as weight $\left.(\mathrm{kg}) /[\text { height }(\mathrm{m})]^{2}\right)$, American Society of Anesthesiologists score, length of operation, performed procedures, and size of largest residual disease were recorded and extracted for data analysis. The complexity of the procedure was quantified using the surgical complexity score previously developed and validated. ${ }^{7}$ Scores ranging from 1 to 3 were assigned for specific surgical procedures based on the complexity of the procedure and a nominal scale stratifies cases into low, intermediate, or high complexity groups.

Hypothermia was defined as a core body temperature lower than $36^{\circ} \mathrm{C}$ at the time of closure of the abdominal incision. Temperature was monitored through an esophageal probe during all procedures. Early postoperative morbidity was the primary outcome in this study and was defined as the occurrence of a listed complication occurring within 30 days of surgery. These complications included mortality; infectious complications including wound infection, pneumonia, or other documented infections within the perioperative period; venous thromboembolic (VTE) events including deep venous thrombosis and pulmonary embolism; cardiac events such as myocardial infarction or arrhythmias requiring treatment; bleeding diathesis; and anastomotic leak. Blood transfusion during the perioperative period was also recorded. Secondary outcomes included 5-year overall survival and disease-free survival. Survival data were ascertained from follow-up data extracted from electronic medical records at the Mayo Clinic, the Mayo Clinic Cancer Center Registry Database, and correspondence from referral institutions.

Continuous data with normal distribution are presented as mean (standard deviation); median and range are used for skewed data. Categorical data are presented as number and percent of patients. Baseline characteristics of treatment cohorts were compared using the $t$ test or Wilcoxon rank sum (Mann-Whitney) test for continuous variables according to the distribution, and $\chi^{2}$ test or Fisher exact test were used for comparison of categorical variables as appropriate. For statistical purposes, variables were stratified in the following manner into two groups which included age ( 75 years or older compared with younger than 75 years); American Society of Anesthesiologists status (American Society of Anesthesiologists 1 or 2 compared with American Society of Anesthesiologists 3 or 4 ); operative time (4 hours or less compared with more than 4 hours); BMI (obese [BMI higher than 30] compared with nonobese); and residual disease $(1 \mathrm{~cm}$ or less compared with more than $1 \mathrm{~cm}$ ). Complexity of surgery was used in the analyses as a nominal variable (low, intermediate, or high surgical complexity). A collective end point including all postoperative complications was the primary end point of the study. Logistic regression models were used to adjust for 
known confounders including age, BMI, preoperative albumin level, length of operation, surgical complexity, and the American Society of Anesthesiologists score. Kaplan-Meier survival curves were used to compare the time to event (death or recurrence) between a patient with hypothermia and a patient with no hypothermia. Differences were considered statistically significant at $P<.05$. All statistical analyses were performed using JMP 8.0.

\section{RESULTS}

One hundred forty-six patients met criteria for inclusion. The mean age was $63.9 \pm 11.7$ years; 46 (31.5\%) patient had a BMI higher than 30; $99(68 \%)$ were stage IIIC and $47(32 \%)$ were stage IV; $84(57 \%)$ were classified as American Society of Anesthesiologists score of 3 or 4 ; and mean operative time was $239 \pm 85$ minutes. Bowel resection was performed in 29 (19\%) of cases, and overall $81 \%$ of the patients had high or intermediate surgical complexity score, which is consistent with an aggressive surgical approach in advanced stage disease. Eighty-one patients $(55 \%)$ had end operative temperature of less than $36^{\circ} \mathrm{C}$. The mean end operative temperature in the hyperthermia group was $36.5 \pm 0.38^{\circ} \mathrm{C}$ with a median of $36.5^{\circ} \mathrm{C}$ (range, $36-37.5^{\circ} \mathrm{C}$ ) compared with $35.2 \pm 0.63^{\circ} \mathrm{C}$ with a median of $35.3^{\circ} \mathrm{C}$ (range $33.4-35.9^{\circ} \mathrm{C}$ ) in the hypothermic group. Variables for normothermic and hypothermic cases are shown in Table 1 and groups were comparable except for a higher percentage of cases older than 75 years old in the hypothermic group.

Early postoperative complications occurred in 45 $(30.8 \%)$ of the included patients. Early complications of any type occurred in $34(42.0 \%)$ of patients in the hypothermic group compared with $11(16.9 \%)$ of normothermic patients with an unadjusted OR of 3.55 (95\% confidence interval [CI $] 1.66-8.07)$ and an adjusted OR of 3.4 (95\% CI 1.48-8.33). Hypothermia, age, and American Society of Anesthesiologists score were all independently associated with early complications, whereas other potential confounding factors such as BMI, length of operation, and surgical complexity did not appear to be associated with postoperative complications. Preoperative albumin was recorded in 50 of $146(34.2 \%)$ with a mean of $3.4 \pm 0.7 \mathrm{~g} / \mathrm{dL}$ with a median of $3.4 \mathrm{~g} / \mathrm{dL}$ (range $2.0-4.4$ ) with no difference in percent of patients with albumin less than $3 \mathrm{~g} / \mathrm{dL}$ between the patients who had end operative temperatures lower than $36^{\circ} \mathrm{C}$ and higher than $36^{\circ} \mathrm{C}$. After adjustment for albumin level, end operative temperature less than $36^{\circ} \mathrm{C}$ remained significantly associated with early complications when
Table 1. Demographic and Baseline Characteristics of the Study Population

\begin{tabular}{|c|c|c|c|}
\hline Variable & $\begin{array}{l}\text { Hypothermic } \\
\qquad(n=81)\end{array}$ & $\begin{array}{l}\text { Normothermic } \\
\qquad(n=65)\end{array}$ & $P$ \\
\hline Age 75 y or older & $21(25.9)$ & $8(12.3)$ & .040 \\
\hline $\begin{array}{l}\text { Body mass index } \\
\text { higher than } 30 \\
\mathrm{~kg} / \mathrm{m}^{2}\end{array}$ & $21(25.9)$ & $25(38.5)$ & .105 \\
\hline ASA score of 3 or 4 & $49(60.5)$ & $35(53.8)$ & .419 \\
\hline $\begin{array}{l}\text { Operative time longer } \\
\text { than } 4 \mathrm{~h}\end{array}$ & $36(44.4)$ & $24(36.9)$ & .359 \\
\hline \multicolumn{4}{|l|}{ Surgical stage } \\
\hline IV & $29(35.8)$ & $18(27.7)$ & .297 \\
\hline IIIC & $52(64.2)$ & $47(72.3)$ & \\
\hline $\begin{array}{l}\text { Cytoreduction to no } \\
\text { visible } \\
\text { residual } \\
\text { disease }\end{array}$ & $19(23.5)$ & $20(30.8)$ & .321 \\
\hline $\begin{array}{l}\text { Cytoreduction to } \\
\text { residual } \\
\text { disease } 1 \mathrm{~cm} \\
\text { or less }\end{array}$ & $59(72.8)$ & $48(73.8)$ & .891 \\
\hline 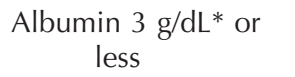 & $12 / 27(44.4)$ & $6 / 23(26.1)$ & .178 \\
\hline \multicolumn{4}{|l|}{$\begin{array}{l}\text { Surgical complexity } \\
\text { score }\end{array}$} \\
\hline Low & $14(17.3)$ & $14(21.5)$ & \\
\hline Intermediate & $51(63)$ & $35(53.8)$ & .538 \\
\hline High & $16(19.8)$ & $16(24.6)$ & \\
\hline
\end{tabular}

ASA, American Society of Anesthesiologists.

Data are $\mathrm{n}(\%)$ or $\mathrm{n} / \mathrm{N}(\%)$ unless otherwise specified.

* Albumin level not available for all patients.

both variables were included in multivariate model with an adjusted OR for end operative temperature less than $36^{\circ} \mathrm{C}$ of $6.2(95 \%$ CI $1.3-45.2, P=.024)$. Serum albumin was not available for the majority of the patients so we were unable to include it in the adjusted models (Table 2).

There were five $(3.4 \%)$ deaths within the first 30 postoperative days. All of these cases had end operative temperature of less than $36^{\circ} \mathrm{C}$. Infectious morbidity was significantly increased in hypothermic patients with an unadjusted OR of 3.19 (95\% CI 1.08-11.71); however, after adjustment, this was not significant with an adjusted OR of 2.99 (95\% CI 0.97-11.35). Hypothermic patients had a higher likelihood of VTE events with an unadjusted OR of 4.32 (95\% CI 1.33-19.37) and adjusted OR of 3.53 (95\% CI 1.02-16.44). Hypothermic patients were more likely to received more than 2 units of blood transfusion with an unadjusted OR of 4.09 (95\% CI 2.078.31 ) and an adjusted OR of 4.79 (95\% CI 2.22 10.81). For many rare individual complications, there was no significant increased risk in the hypothermia cases. The actual rates of anastomotic leak $(2.7 \%$ 
Table 2. Association Between Hypothermia and Other Risk Factors and the Development of Any Early Postoperative Complication

\begin{tabular}{|c|c|c|c|c|c|c|}
\hline \multirow[b]{2}{*}{ Variable } & \multicolumn{3}{|c|}{ Univariable } & \multicolumn{3}{|c|}{ Multivariable } \\
\hline & OR & $95 \% \mathrm{Cl}$ & $P$ & OR & $95 \% \mathrm{Cl}$ & $P$ \\
\hline $\begin{array}{l}\text { Hypothermia (end of operation } \\
\text { temperature less than } 36^{\circ} \mathrm{C} \text { ) }\end{array}$ & 3.55 & $1.66-8.07$ & .002 & 3.35 & $1.45-8.28$ & .006 \\
\hline Age 75 y or older & 4.50 & $1.93-10.79$ & $<.001$ & 4.50 & $1.72-12.23$ & .003 \\
\hline BMl higher than $30 \mathrm{~kg} / \mathrm{m}^{2}$ & 1.31 & $0.61-2.74$ & .48 & 1.67 & $0.66-4.24$ & .28 \\
\hline ASA score & 3.15 & $1.48-7.16$ & .004 & 2.71 & $1.14-6.84$ & .029 \\
\hline Operative time longer than $4 \mathrm{~h}$ & 1.07 & $0.52-2.18$ & .85 & 1.31 & $0.56-3.12$ & .54 \\
\hline Surgical complexity score* & & & .65 & & & .97 \\
\hline Intermediate & 0.82 & $0.34-2.07$ & & 1.06 & $0.34-3.38$ & \\
\hline High & 0.60 & $0.19-1.82$ & & 0.93 & $0.24-3.64$ & \\
\hline
\end{tabular}

OR, odds ratio; $\mathrm{Cl}$, confidence interval; BMI, body mass index; ASA, American Society of Anesthesiologists.

* Surgical complexity score included three levels of complexity (low, intermediate, and high) based on previously published data. ${ }^{7}$

compared with $0.7 \%)$, cardiovascular events $(4.1 \%$ compared with $0.7 \%)$, readmission (3.4\% compared with $0.7 \%)$, and reoperation (4.8\% compared with $0.7 \%)$ were all higher in the hypothermia group compared with the normothermia group (Table 3). Of those rare complications, only reoperation was significantly correlated to hypothermia after adjustment for other known confounders with an adjusted OR of $4.96(95 \%$ CI $0.80-95.7)$.

Finally, we observed a decreased 5-year overall survival in the hypothermic patient with a median overall survival of 34 months compared with 45 month $(P=.045)$ when considering all patients (Fig. 1). In the optimally resected to less than 1 -cm subgroup of cases, the difference in overall survival remained significant with a median overall survival of 40 months compared with 48 months $(P=.049)$ (Fig. 2A). This survival difference was not observed in the subgroup of patients in whom optimal debulking of less than $1 \mathrm{~cm}$ was not achieved (Fig. 2B).

\section{DISCUSSION}

Although hypothermia has been studied in other surgical disciplines, its role in gynecologic surgery has not been previously described. The present study shows that intraoperative hypothermia is a significant and independent risk factor for early postoperative complications and early mortality in women undergoing ovarian cancer cytoreductive surgery. Women with intraoperative hypothermia are three times more likely to experience a postoperative complication compared with women who maintained normal temperature during the operation. Hypothermic patients also appear to be significantly more likely to experience VTE events and infectious complications. All five patients in our cohort who died within the first 30 days after surgery had an intraoperative temperature of less than $36^{\circ} \mathrm{C}$. These findings suggest that there is a strong association between end operative temperature and morbidity and mortality. Given the modifiable nature of in-

Table 3. Association Between Hypothermia and Individual Early Postoperative Complications

\begin{tabular}{|c|c|c|c|c|c|c|}
\hline Outcome & Total $(n=146)$ & Hypothermic $(n=81)$ & Normothermic $(n=65)$ & OR & $\begin{array}{c}\text { Univariable } \\
95 \% \mathrm{Cl}\end{array}$ & $P$ \\
\hline Death with $30 \mathrm{~d}$ & $5(3.6)$ & $5(6.2)$ & $0(0.0)$ & 9.42 & $0.51-173.55$ & * \\
\hline Infectious complication & $18(12.3)$ & $14(17.3)$ & $4(6.2)$ & 3.19 & $1.08-11.71$ & .05 \\
\hline VTE event & $17(11.6)$ & $14(17.3)$ & $3(4.6)$ & 4.32 & $1.33-19.37$ & .027 \\
\hline Cardiac event & $7(4.8)$ & $6(7.4)$ & $1(1.5)$ & 5.12 & $0.84-97.90$ & .14 \\
\hline Bowel leak & $5(3.4)$ & $4(4.9)$ & $1(1.5)$ & 3.32 & $0.48-65.87$ & .29 \\
\hline Readmission & $6(4.1)$ & $5(6.1)$ & $1(1.5)$ & 4.21 & $0.66-81.73$ & .19 \\
\hline Reoperation & $8(5.5)$ & $7(8.6)$ & $1(1.5)$ & 6.05 & $1.04-114.72$ & .10 \\
\hline Transfusion more than 2 units $^{\dagger}$ & $79(54.1)$ & $56(69.1)$ & $23(35.4)$ & 4.09 & $2.07-8.31$ & $<.001$ \\
\hline
\end{tabular}

OR, odds ratio; $\mathrm{Cl}$, confidence interval; VTE, venous thromboembolic.

Data are $\mathrm{n}(\%)$ unless otherwise specified.

* Unable to estimate a $P$ value as a result of the lack of deaths in the normothermic group.

${ }^{+}$Blood transfusion was not considered one of the early complications included in the combined primary end point. 




Fig. 1. Overall survival in hypothermic compared with normothermic patients.

Moslemi-Kebria. Hypothermia and Perioperative Morbidity. Obstet Gynecol 2012.

traoperative normothermia, this should be the focus of future studies to improve outcomes after radical surgery for ovarian cancer.

Kruz and colleagues in a prospective study in patients undergoing colorectal surgery showed that incidence of postoperative surgical wound infection after colon resection increases three times if the core body temperature was reduced by $1.9^{\circ} \mathrm{C} .^{2}$ Infectious complications after major abdominal surgery could range from wound infection to intraperitoneal abscess formation and sepsis. Infectious complications prolong hospital stay, increase costs, and often result in delay of adjuvant chemotherapy. Hypothermia alters immune function and may delay wound healing and facilitate infectious processes. Studies have shown that hypothermia impairs immunity by affecting natural killer cell and cell-mediated antibody functions. ${ }^{8} \mathrm{Hy}-$ pothermia has been associated with suppressed phagocytic activity, decreased migration of polymorphonuclear cells, and reduced oxidative bacterial killing by neutrophils. ${ }^{9}$ Another indirect way through which intraoperative hypothermia may facilitate infection is related to thermoregulatory vasoconstriction and subsequent tissue hypoxia. ${ }^{10,11}$

Increased metabolic demand and oxygen consumption resulting from shivering ${ }^{12}$ and cold-induced sympathoadrenal activation have been suggested to predispose patients to myocardial ischemia. ${ }^{13}$ A prospective, randomized study in patients undergoing major cardiovascular surgery demonstrated that reduction of core body temperature of only $1.3^{\circ} \mathrm{C}$ led to a threefold increase in the rate of perioperative morbid cardiac events such as ischemia and ventricular

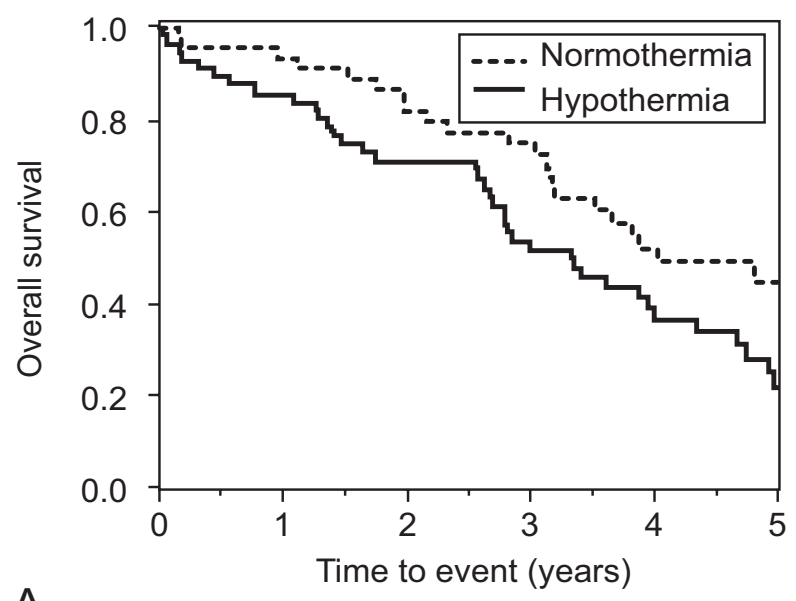

A

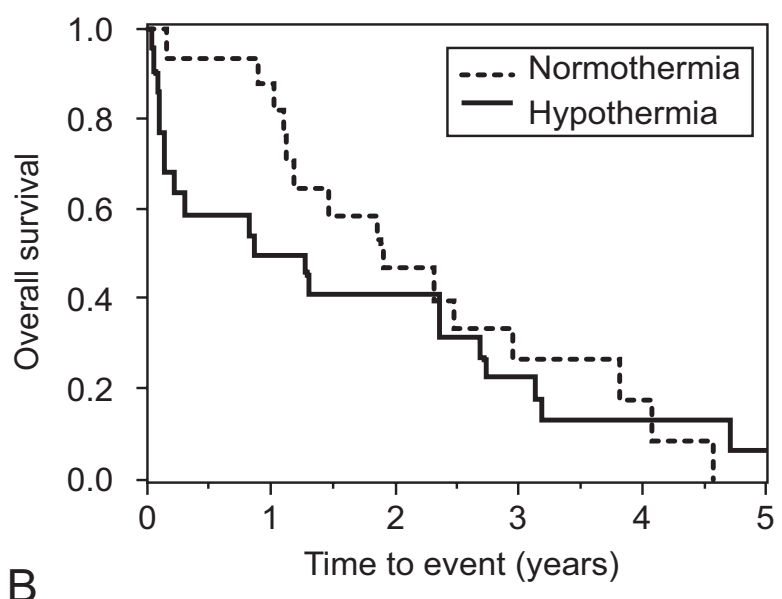

Fig. 2. Overall survival in hypothermic compared with normothermic patients in A. a group with optimal cytoreduction to less than $1 \mathrm{~cm}$ and B. a suboptimally cytoreduced group.

Moslemi-Kebria. Hypothermia and Perioperative Morbidity. Obstet Gynecol 2012.

tachycardia. ${ }^{4}$ In our study, after adjusting for other factors, the association between hypothermia and cardiovascular events just exceeded statistical significance (OR 6.06, $P=.056$ ) in patients undergoing laparotomy. Remembering that this is traditionally a low-risk population for cardiac events, the data are strongly suggestive of a similar effect of hypothermia on cardiac events in patients with ovarian cancer as reported for other surgical cases.

Hypothermia promotes abnormal platelet aggregation and this may explain the increased rate of VTE events seen in hypothermic patients in our cohort. Consistent with this, we also observed an increased rate of perioperative blood transfusion in hypothermic patients. Other studies have shown that hypothermia can reversibly impair platelet activation and 
decrease generation of thrombin; this subsequently leads to coagulopathy and increased intraoperative blood loss. ${ }^{14,15}$ Hypothermia also inhibits enzymatic reaction of the coagulation cascade, resulting in prolongation of prothrombin time and partial thromboplastin time at hypothermic temperatures. ${ }^{15}$ Because coagulation testing is performed at $37^{\circ} \mathrm{C}$, rather than at the patient's actual in vivo temperature, generally the contribution of hypothermia to objective hemorrhagic diathesis can be missed in the clinical setting. A study by Schmeid and colleagues demonstrated that a reduction of just $1.6^{\circ} \mathrm{C}$ in core temperature increases blood loss by $500 \mathrm{~mL}$ and significantly increases transfusion in patients undergoing hip arthroplasty. ${ }^{3}$ Our data are consistent with this observation.

We observed an increased rate of overall early postoperative complications and perioperative mortality in hypothermic patients. In fact, in this relatively homogeneous group of patients with advanced ovarian cancer undergoing moderate to high complexity surgery, hypothermia was an independent predictor for the development of a perioperative complication. The cost-effectiveness of maintaining normothermia in patients undergoing colorectal surgery and hip arthroplasty has been shown in prospective studies.,3 Similar results can be expected in patients with ovarian cancer given the present study. Additionally, postoperative complications may delay the start of adjuvant chemotherapy, which can subsequently lead to inferior oncologic outcomes. Some authors suggested enhanced angiogenesis and immunosuppression as potential factors that facilitate cancer cell seeding and metastasis in the perioperative period. ${ }^{16}$ In our patient series, we observed a significant association between intraoperative hypothermia and overall survival. Whether this is 1) primarily a result of the early mortality in five cases; 2) because of a delay in chemotherapy in hypothermic cases; or 3) a reflection that patients who develop hypothermia may have poor performance status or more tumor burden is not clear.

Several measurements to maintain core body temperature during surgery have been advocated. These include forced air warmers, fluid warmers, and heated mats. Newer methods including autoregulated heating based on the actual body temperature has been proposed. In addition, perioperative skin warming has been shown to reduce the initial postinduction hypothermia, intraoperative hypothermia, and postoperative shivering, even for procedures lasting for more than 3 hours. Furthermore, a single hour of preoperative skin surface warming has been reported to reduce the rate at which core hypothermia devel- oped during the first hour of anesthesia. Irrespective of the method, in patients undergoing total hip arthroplasty, even a small difference in median core intraoperative temperature of $0.5^{\circ} \mathrm{C}$ resulted in significantly less blood loss among the patients who were warmed. . $^{1718}$

Although limited by sample size, we did not find a correlation between operative times and hypothermia suggesting that other factors are probably more important. This is important given the oncologic benefit from more complex surgical procedures to render lowest residual disease. ${ }^{7}$ In addition to sample size, our study was limited by using only end operating temperature to define hypothermia, as is currently defined. We anticipate more data from the literature regarding the most appropriate measurement of hypothermia during long surgical cases. The study was limited by the lack of direct information about coagulopathy; however, blood transfusion was reported and can be used as a surrogate for coagulation defects. The study was also limited by the lack of full recording of albumin preoperatively in all patients; this prevented full evaluation of albumin levels in the final multivariate model. Nevertheless, end operative temperature less than $36^{\circ} \mathrm{C}$ remained significantly associated with early complications after adjustment for albumin. Finally, the overall survival analysis was limited by relatively small size precluding use of Cox proportional hazard modeling to adjust for known risk factors, including age, chemotherapy type, and time from surgery to start of antibiotics. Nevertheless, a subgroup analysis based in the subgroup of patient who had optimal debulking.

This study addresses the role of hypothermia as a risk factor for postoperative complications in women undergoing cytoreductive surgery for ovarian cancer. Our data demonstrate an association between intraoperative hypothermia and short-term preoperative complications and early mortality after ovarian cancer cytoreductive surgery. Whether hypothermia is causal or merely a sign of endogenous patient comorbidities remains to be answered with prospective studies controlling intraoperative temperature. However, this is a critically important observation because temperature is a modifiable factor, which could result in reduced morbidity. Our data suggest active warming methods and monitoring to maintain intraoperative temperature above $36^{\circ} \mathrm{C}$ is reasonable and may result in decreased morbidity and cost.

\section{REFERENCES}

1. Xiong J, Kurz A, Sessler DI, Plattner O, Christensen R, Dechert $\mathrm{M}$, et al. Isoflurane produces marked and nonlinear 
decreases in the vasoconstriction and shivering thresholds. Anesthesiology 1996;85:240-5.

2. Kurz A, Sessler DI, Lenhardt R. Perioperative normothermia to reduce the incidence of surgical-wound infection and shorten hospitalization. Study of Wound Infection and Temperature Group. N Engl J Med 1996;334:1209-15.

3. Schmied H, Kurz A, Sessler DI, Kozek S, Reiter A. Mild hypothermia increases blood loss and transfusion requirements during total hip arthroplasty. Lancet 1996;347:289-92.

4. Frank SM, Fleisher LA, Breslow MJ, Higgins MS, Olson KF, Kelly S, et al. Perioperative maintenance of normothermia reduces the incidence of morbid cardiac events. A randomized clinical trial. JAMA 1997;277:1127-34.

5. Aletti GD, Dowdy SC, Gostout BS, Jones MB, Stanhope RC, Wilson TO, et al. Quality improvement in the surgical approach to advanced ovarian cancer: the Mayo Clinic experience. J Am Coll Surg 2009;208:614-20.

6. von Elm E, Altman DG, Egger M, Pocock SJ, Gotzsche PC, Vandenbroucke JP. The Strengthening the Reporting of Observational Studies in Epidemiology (STROBE) statement: guidelines for reporting observational studies. Lancet 2007; 370:1453-7.

7. Aletti GD, Dowdy SC, Podratz KC, Cliby WA. Relationship among surgical complexity, short-term morbidity, and overall survival in primary surgery for advanced ovarian cancer. Am J Obstet Gynecol 2007;197:676.e1-7.

8. Beilin B, Shavit Y, Razumovsky J, Wolloch Y, Zeidel A, Bessler H. Effects of mild perioperative hypothermia on cellular immune responses. Anesthesiology 1998;89:1133-40.

9. Wenisch C, Narzt E, Sessler DI, Parschalk B, Lenhardt R, Kurz A, et al. Mild intraoperative hypothermia reduces production of reactive oxygen intermediates by polymorphonuclear leukocytes. Anesth Analg 1996;82:810-6.

10. Doufas AG. Consequences of inadvertent perioperative hypothermia. Best Pract Res Clin Anaesthesiol 2003;17:535-49.

11. Greif R, Akça O, Horn EP, Kurz A, Sessler DI; Outcomes Research Group. Supplemental perioperative oxygen to reduce the incidence of surgical-wound infection. N Engl J Med 2000;342:161-7.

12. Bilotta F, Pietropaoli P, La Rosa I, Spinelli F, Rosa G. Effects of shivering prevention on haemodynamic and metabolic demands in hypothermic postoperative neurosurgical patients. Anaesthesia 2001;56:514-9.

13. Frank SM, Higgins MS, Breslow MJ, Fleisher LA, Gorman RB, Sitzmann JV, et al. The catecholamine, cortisol, and hemodynamic responses to mild perioperative hypothermia. A randomized clinical trial. Anesthesiology 1995;82:83-93.

14. Michelson AD, MacGregor H, Barnard MR, Kestin AS, Rohrer MJ, Valeri CR. Reversible inhibition of human platelet activation by hypothermia in vivo and in vitro. Thromb Haemost 1994;71:633-40.

15. Rohrer MJ, Natale AM. Effect of hypothermia on the coagulation cascade. Crit Care Med 1992;20:1402-5.

16. Shakhar G, Ben-Eliyahu S. Potential prophylactic measures against postoperative immunosuppression: could they reduce recurrence rates in oncological patients? Ann Surg Oncol 2003;10:972-92

17. De Witte JL, Demeyer C, Vandemaele E. Resistive-heating or forced-air warming for the prevention of redistribution hypothermia. Anesth Analg 2010;110:829-33.

18. Sajid MS, Shakir AJ, Khatri K, Baig MK. The role of perioperative warming in surgery: a systematic review. Sao Paulo Med J 2009;127:231-7.

\section{Rapid Review Process at Obstetrics \& Gynecology}

At Obstetrics \& Gynecology, most authors receive their first decision (reject or revise) within 30 days of submission. Our online submission process also enables most authors to submit and publish a manuscript within 6 months or less. Between December 2010 and December 2011, an average of $87 \%$ of manuscripts were published within 6 months or less.

To take advantage of the rapid review process and submit your research, please visit http://ong.editorialmanager.com. 